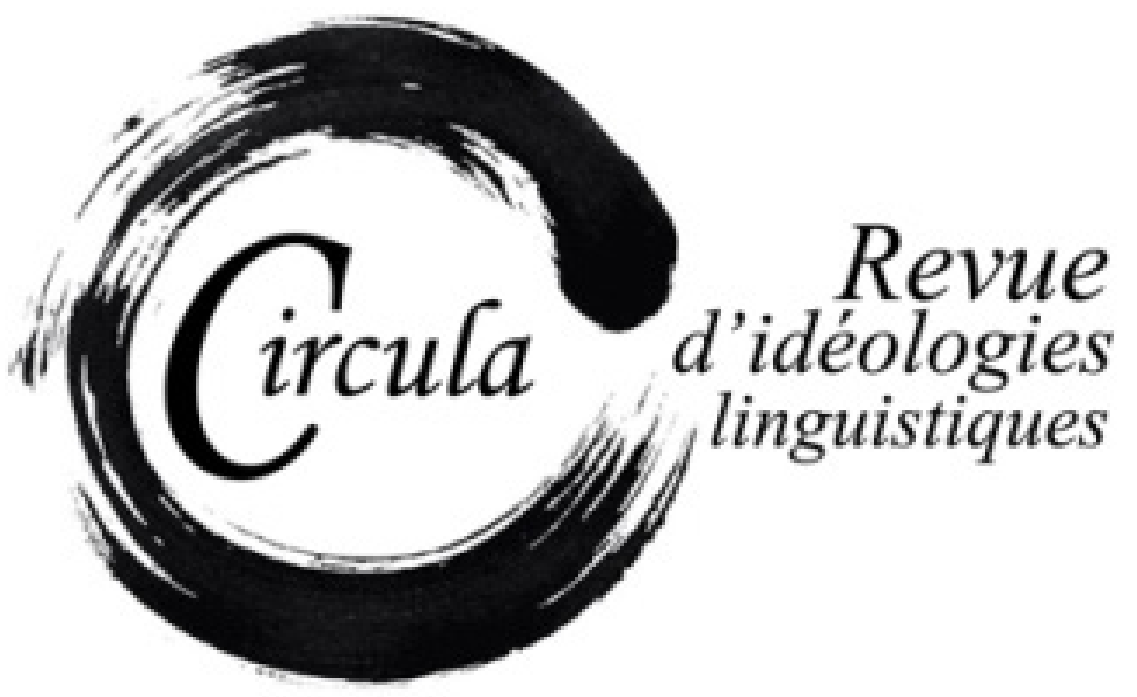

TITRE: LES EXPÉRIENCES ARTISTIQUES EN CONTEXTE PLURILINGUE : UNE MISE À L'ÉPREUVE DE LA VULNÉRABILITÉ LINGUISTIQUE?

Auteur: Maud SÉrusclat-Natale, Université Paul Valéry Montpellier III

Revue: CIRCula, NumÉro 12 : LA VULNÉRABILITÉ LINGUISTIQUE

Directrice: Claudia Torres CAStillo

PAGES: $107-131$

ISSN: 2369-6761

URI: HTTP://HDL.HANDLE.NET/11143/18445

DOI: HTTPS://DOI.ORG/10.17118/11143/18445 


\title{
Les expériences artistiques en contexte plurilingue : une mise à l'épreuve de la vulnérabilité linguistique?
}

Maud Sérusclat-Natale, Université Paul Valéry Montpellier III m.natale@mascene.eu

Résumé : «Parlemonde », festival artistique, a réuni 15 artistes et 250 participants plurilingues, majoritairement récemment arrivés en France. Né d'une collaboration entre une scène nationale et l'école, «Parlemonde » fait de la diversité linguistique et culturelle d'un territoire un catalyseur de création. Si les pratiques artistiques sont encouragées pour l'appropriation d'une nouvelle langue, elles demeurent à l'école considérées comme une épreuve risquée, une mise en danger ou une (sur) exposition de ces élèves a priori vulnérables du fait de leur qualité d'apprenants de français et de leur expérience de l'exil. Nous montrerons qu'elles révèlent les processus de construction des situations de vulnérabilité (linguistique, culturelle, scolaire) et qu'elles contribuent, à certaines conditions, à les désamorcer en légitimant les répertoires langagiers et en créant, par l'exposition publique, les conditions de dépassement du sentiment d'insécurité linguistique et culturelle, transcendant la vulnérabilité en une reconnaissance commune des forces de chacun (adolescents, enseignants, artistes et publics).

Mots-clés : Élève allophone arrivant ; représentations ; expériences artistiques ; vulnérabilité ; appropriation

\begin{abstract}
Parlemonde", an artistic festival, brought together 15 artists and 250 multilingual participants, most of whom had recently arrived in France. Born from a collaboration between a national theater and public school, "Parlemonde" makes the linguistic and cultural diversity of a territory a catalyst for creation. Although artistic practices for the appropriation of a new language are encouraged, in school they are still considered a risky ordeal and an endangerment or (over)exposure of these seemingly vulnerable students, due to their status as learners and their experience of exile. We will demonstrate, on the one hand, that they expose the processes of construction of situations of vulnerability (linguistic, cultural, academic) and that they contribute, under certain conditions, to their deconstruction by legitimizing the languages and by creating, through public exposure, the conditions to overcome the feeling of linguistic and cultural insecurity; transcending vulnerability into a common recognition of each person's strengths (teenagers, teachers, artists and audiences).
\end{abstract}

Key words: newly arrived pupil; representation; artistic experiences; vulnerability; appropriation 


\section{Introduction}

Cette contribution repose sur notre recherche doctorale en cours, portant sur le festival Parlemonde se déroulant en biennale depuis 2017 à Montbéliard (France). Organisé par une scène nationale publique nommée «MA scène nationale ${ }^{1}$ » (désormais MA) en collaboration avec le Rectorat de l'académie de Besançon, particulièrement avec les services dédiés à l'action culturelle DAAC ${ }^{2}$ et le CASNAV ${ }^{3}$, Parlemonde est un programme de résidences d'artistes qui ont lieu majoritairement en milieu scolaire, et qui entend favoriser l'appropriation de la langue française et l'inclusion des élèves allophones nouvellement arrivés (désormais EANA). Il se fonde sur l'idée que les plurilinguismes de tous (artistes, participants, enseignants, etc.) peuvent être un moteur de création artistique, de rencontre et de reconnaissance sociale.

Nous présenterons d'abord en quoi les EANA rencontrés lors de notre recherche et principaux participants de Parlemonde, sont parfois considérés comme « vulnérables » ou en situation de vulnérabilité linguistique, scolaire et plus largement sociale, par les adultes collaborant avec eux. En effet, «barrière de la langue », fragilité psychologique ou traumatismes liés à l'exil (Mestre 2015 ; OCDE 2008) semblent enfermer les EANA dans un continuum de l'empêchement que l'urgence de la diplomation et la nécessité de rentabilité scolaire visant l'insertion professionnelle viennent renforcer. Nous analyserons ces obstacles systémiques souvent avancés pour justifier le fait que leur participation à un projet artistique public constitue un risque qui les surexpose, et qui pourrait accroitre leur vulnérabilité. Nous montrerons au contraire que les expériences artistiques conçues en partenariat sont vectrices d'empowerment (Sen, 2009) et transforment tant ceux qui les exercent que ceux qui les reçoivent. Notre approche, ethnographique, reposera principalement sur les outils de l'analyse du discours. Nous utiliserons un corpus d'entretiens semi-directifs, nos notes de terrain et des exemples de créations artistiques de Parlemonde pour expliquer comment ce type d'actions peut contribuer, à certaines conditions, à favoriser l'entrée dans la langue de scolarisation pour les EANA impliqués, mais également à valoriser les plurilinguismes devenant vecteurs de cohésion sociale, réveillant un patrimoine commun dans un territoire riche d'une histoire migratoire ancienne relativement ignorée.

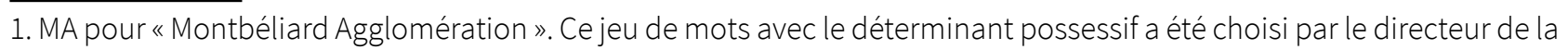
structure pour renforcer l'idée centrale de son projet qui vise à ce que les habitants s'approprient ce lieu de culture dédié à la création artistique contemporaine. Sa labellisation par l'État remonte à plus de 20 ans.

2. DAAC pour Délégation académique à l'action culturelle.

3. CASNAV pour Centre académique pour la scolarisation des enfants nouvellement arrivés et/ou issus de familles itinérantes ou de voyageurs. 


\section{2. (Dé)composer les situations de vulnérabilité}

Étymologiquement, la vulnérabilité est inhérente à la condition humaine, et signifie être exposé au risque de blessure. La catégorie de la vulnérabilité est de plus en plus utilisée en sciences sociales et humaines, ainsi qu'en droit. Le substantif « vulnérabilité » renvoie alors à un groupe humain hétéro-déterminé, repéré comme potentiellement plus fragile, en fonction d'un contexte ou d'un environnement donné. Le Conseil de l'Europe (COE), dans le but de construire des politiques communes de prévention, a désigné 3 groupes sociaux comme spécifiquement exposés à la vulnérabilité : les personnes en situation de handicap, les migrants et les enfants. Dans les travaux des Nations Unies, ces 3 groupes sont rejoints par les femmes et les populations autochtones ${ }^{4}$ tandis que dans les représentations sociales communément partagées, en particulier en temps de crise, sont vulnérables les jeunes (inexpérimentés et/ou imprudents), les plus anciens (fragilisés par l'âge) et les personnes économiquement précaires. Dans cette approche, l'adjectif « linguistique » adossé à vulnérabilité, renverrait au fait qu'un sujet ne soit pas en pleine capacité de communiquer et de s'exprimer avec le groupe dans lequel il est intégré. Sa vulnérabilité ne serait pas l'une de ses qualités propres, mais bien relative à un état lié à une situation vécue, ce qu'Ennuyer précise ainsi : «La vulnérabilitë d'un individu [est] occasionnée par l'interaction entre une personne et son environnement au sens large » (Ennuyer, 2017 : 371). On pourrait aller plus loin en ajoutant que la vulnérabilité linguistique d'un individu pourrait être occasionnée également par l'impossibilité (vécue, ressentie ou imaginée) de l'interaction avec cet environnement. Dans une société ou la « maitrise de la langue » est à la fois un moyen d'intégration et une condition de sélection pour l'octroi d'un titre de séjour par exemple, les populations allophones et déplacées sont désignées par les institutions comme vulnérables et le facteur linguistique est central : à la fois symptôme et cause de leur vulnérabilité sociale. En France comme ailleurs, nous nous situons dans un contexte où les institutions s'emparent de ce concept de vulnérabilité au titre de l'inclusion. Le projet de création artistique que nous analysons en fait dialoguer 2 : l'école et une structure culturelle publique. Elles partagent une mission de service public: l'éducation artistique et culturelle. Elles portent une attention particulière aux nouveaux arrivants, qu'elles désignent cependant de manière différente (nous y reviendrons) et elles se mobilisent pour être accessibles. Ce paradigme de l'accessibilité, ou de l'accessibilisation (Ebersold et ArmagnagueRoucher, 2017), est le pendant d'une politique d'inclusion et revêt différents aspects au cœur desquels la question des représentations est cruciale.

4. Information disponible sur http://www.claiminghumanrights.org/special_groups.html?L=1. [Page consultée le 2 octobre 2020.] 


\subsection{Les EANA : des apprenants vulnérables?}

En France, la scolarisation est un droit pour tous les enfants de 3 à 18 ans et l'instruction est obligatoire jusqu'à la majorité ${ }^{5}$ quelle que soit la situation légale, sociale ou linguistique de l'élève (et/ou de sa famille) sur le territoire. Depuis le Bulletin officiel (B.O.) de 2012, les modalités de scolarisation des EANA se font dans une visée inclusive, au moyen de dispositifs d'enseignement spécifiques appelés UPE2A (Unités pédagogiques pour les élèves allophones arrivants) ouverts sur la classe dite " ordinaire » et pilotés dans chaque région académique par les CASNAV. Les textes prévoient que les élèves soient inscrits selon leur âge dans la classe qui correspond. Ils y suivent certains cours selon leurs compétences et la quittent ponctuellement pour rejoindre l'UPE2A afin de bénéficier de cours spécifiques de français langue de scolarisation, et/ou de remise à niveau en diverses matières comme les mathématiques, l'anglais, les sciences, etc. Chaque élève accueilli a été préalablement évalué avec l'aide des CASNAV et bénéficie d'un emploi du temps individualisé pour répondre à ses «besoins éducatifs particuliers».

Dans les différents textes régissant cette organisation systémique, le terme de "vulnérabilité » n'apparait pas, au profit d'une politique de prise en compte des « besoins éducatifs particuliers » et de compensation faisant par exemple échos aux travaux du comité interministériel du handicap et de l'OCDE. Cette dernière catégorise 3 types de situations de besoins éducatifs particuliers : la déficience pour « les élèves présentant des déficiences ou incapacités » liées à des troubles médicaux; la difficulté pour les élèves présentant des « troubles du comportement ou des troubles affectifs et des apprentissages » desquels résultent des « problèmes d'interaction avec l'environnement éducatif » et les « désavantages », pour les élèves défavorisés en raison de «facteurs socio-économiques, culturels et/ou linguistiques, pour lesquels le besoin éducatif consiste à compenser les désavantages liés à ces facteurs » (OCDE, 2008 : 22). Cette dénomination a connu des évolutions mais demeure vivement critiquée par les chercheurs car elle associe de facto la question de l'allophonie à la non-maitrise de la langue de scolarisation et donc à un handicap conduisant à un « risque scolaire ». Cette conception viendrait renforcer la représentation sociale de l'EANA comme apprenant « vulnérable » car l'altérité demeure pensée par les institutions comme une situation de déficit (Métraux, 2011). Cela va à l'encontre de près de 30 ans de recherche sur le bi-plurilinguisme qui ont démontré que parler plusieurs langues, à des degrés divers, n'était pas une situation conduisant systématiquement à un handicap social mais plutôt un avantage cognitif, social et culturel considérable, s'il est correctement accompagné (Billiez, 1985 ; Lüdi et Py 1986 ; Cummins, 2001 ; Auger, 2010). Si le paradigme du « besoin éducatif particulier » reflète une volonté politique d'inclusion des EANA, il suppose une nécessaire adaptation du système scolaire devant s'appuyer sur - et produire - de nouvelles ressources pour scolariser ces publics «spécifiques ». Or, l'élève allophone arrivant en contexte scolaire est très souvent davantage pensé comme en situation de risque d'échec, et ce constat supplante la valorisation de son plurilinguisme et/ou de son bi-plurilinguisme émergeant (García, 2009) en tant que force

5. Information disponible sur https://www.service-public.fr/particuliers/vosdroits/F1898. [Page consultée le 2 octobre 2020.] 
potentielle d'apprentissage de la langue cible. De plus, la prise en compte des besoins particuliers et la mise en place de compensations adaptées ne vont pas de soi. Les difficultés présentées par les enseignants sont très souvent liées à l'évaluation ou à la certification, au motif d'une représentation de l'idéal républicain qui se réclamerait d'une équité de traitement confondue avec un principe d'égalité absolu rendant toute compensation comme une rupture de celle-ci. C'est la trace, consciente ou non, du modèle du creuset républicain et témoigne de la complexité de sa nécessaire transformation (Renaut, 2009). Cela construit des situations de vulnérabilité, pour les élèves, EANA inclus. Pour illustrer cela au niveau institutionnel, citons le rapport intitulé « Les aménagements d'épreuves d'examens pour les élèves et étudiants en situation de handicap » rédigé par l'Inspection générale en 2018. Sont mentionnées dès la page 3 les « difficultés éthiques pour faire respecter le droit, garantir l'équité de traitement et accompagner les jurys sans rompre avec les principes d'indépendance et d'égalité qui contribuent à la valeur du diplôme délivré » (Gavini-Chevet, Delécluse et Bigaut, 2018 : 3). Dans le cas des EANA, aucun aménagement d'épreuve n'est prévu, sauf, depuis 2017, pour l'une des épreuves écrites de la discipline «français » (la rédaction) du diplôme de fin de collège, pour laquelle les textes prévoient l'autorisation de l'usage d'un dictionnaire «éventuellement bilingue »'. Le rapport précise d'ailleurs qu'il y a des cas problématiques:

La même contribution fait état de prescriptions qui manquent de précision ou qui paraissent en dehors du champ règlementaire des attributions des médecins (l'exemple donné est la mise à disposition d'un dictionnaire pour un élève allophone qui n'était pas, par ailleurs, en situation de handicap avéré. (Gavini-Chevet, Delécluse et Bigaut, 2018: 14)

Cet exemple, parmi tant d'autres (Adam-Maillet et Sérusclat-Natale, 2019), témoigne du fait que l'école réactive l'idéologie d'un État monolingue, voire glottophobe et glottophage (Calvet, 1974 ; Blanchet, 2016; Escudé, 2018). Le système scolaire demeure pour les chercheurs « l'un des terrains privilégiés de l'analyse des discriminations linguistiques » selon les termes de Veret (2018: §3) dans son compte-rendu de Langues et discriminations. Il y souligne d'ailleurs que « la plupart des auteurs du numéro décrivent en effet l'école française comme le lieu d'une discrimination linguistique systématisée, qui perpétue "la matrice d'idéologie monolingue" (Escudé, 2018) autour de laquelle s'est forgé l'État-nation » (Veret, 2018 : § 3).

\subsection{Vulnérabilité, risque scolaire ou difficulté?}

Les travaux de recherche qui s'intéressent à la scolarité des EANA montrent que ces derniers sont très souvent associés au paradigme de la "difficulté scolaire », attribuée le plus souvent soit à un

6. Cette situation vient d'évoluer grâce à une note de la Direction Générale de l'enseignement scolaire, envoyée aux recteurs le 2 avril 2021. 
manque de scolarisation antérieure 7 , soit à un déficit de «maitrise de la langue », et parfois aux 2 facteurs. II a été démontré que ces discours reposaient souvent sur un manque de formation des enseignants (Auger, 2010, 2013 ; Goï, 2015), ce que relèvent également Armagnague et al. dans le rapport EVASCOL 2018 commandé par le Défenseur des droits. Ces représentations ont, pour Auger, « un impact majeur sur l'ensemble des partenaires du système scolaire : enseignants, enfants, parents, acteurs sociaux, etc. Elles sont souvent en décalage par rapport aux recherches en (socio)linguistique et en didactique des langues» (Auger, 2008 : 187). Elle ajoute que les discours « soulignent aussi davantage les difficultés que les éléments positifs de leur scolarisation» (Auger, 2010: 17).

Les principes de l'inclusion, inscrits en France dans la loi ${ }^{8}$ définissent un cadre juridique ambitieux, mais la « souplesse » - selon le terme du B.O. de 2012 - nécessaire à leur mise en place, manque sur le terrain. Cela place les EANA ne maitrisant pas encore la langue de scolarisation, en situation de vulnérabilité (linguistique, scolaire, et donc sociale). Ils sont de facto menacés d'exclusion, d'échec, alors même que leurs compétences linguistiques, scolaires, sociales, seraient invisibilisées par le système lui-même. Les sociologues Ebersold et Armagnague-Roucher décrivent ce phénomène ainsi :

La persistance des inégalités rappelle qu'un élève jugé à problème est celui qui pose problème aux acteurs du système éducatif du point de vue social. Les manières de faire et d'être de ceux dérogeant aux normes scolaires et aux exigences habituelles du métier d'élève bousculent les règles, les connaissances, les repères et les usages et situent les possibles scolaires dans le développement de stratégies éducatives complexes. Elles empêchent l'identification de routines permettant de conjuguer les exigences inhérentes à la personnalisation des pratiques aux enjeux identitaires et professionnels que demande la reconfiguration des rapports entre enseignant et élève. Elles remettent en cause l'effet miroir qui traverse toute relation sociale et interrogent les membres de la communauté scolaire dans leurs certitudes, questionnent l'image qu'ils se forgent de leur fonction, de leur rôle et d'eux-mêmes et engendrent des tâtonnements pédagogiques et des attitudes compassionnelles fondées sur les subjectivités individuelles. (Ebersold et Armagnague-Roucher, 2017 : 138)

Cet état des lieux est important à poser pour comprendre les enjeux de Parlemonde. Pour les 7 établissements scolaires impliqués, les objectifs qui figurent dans les « fiches actions ${ }^{9}$ » des projets Parlemonde (documents institutionnels à présenter lors des commissions validant non seulement la pertinence éducative du projet mais aussi les modalités de son financement) on trouve :

7. Voir Eduscol : "L'importance des dynamiques territoriales et d'un travail partenarial afin d'inscrire les élèves les plus vulnérables, notamment ceux qui ont été peu scolarisés antérieurement, dans une logique de parcours de formation, d'inclusion et d'insertion professionnelle », disponible sur https://eduscol.education.fr/cid73170/inclusion-scolaire-des-eleves-allophones-et-des-enfants-de-familles-itinerantes.html) [Page consultée le 2 octobre.]

8. Loi n² 2005-102 du 11 février 2005 pour l'égalité des droits et des chances

9. Ces textes sont co-écrits par les enseignants impliqués dans les projets et la structure culturelle puis visés par les chefs d'établissement. 
- Déségrégation des dispositifs UPE2A du lycée grâce et par le contact des élèves avec l'artiste dans une démarche de création au centre de laquelle on interrogera la question des langues.

- Favoriser l'inclusion et valoriser le plurilinguisme

- $\quad$ Circulation des élèves, dans les apprentissages (FLS et Pédagogie de projet).

- Travail artistique et interdisciplinaire sur la question de la trace, du territoire, et de l'orientation en valorisant le répertoire linguistique des participants et de l'établissement (élèves et équipes), notamment le patrimoine linguistique propre aux ateliers professionnels.

- Favoriser l'engagement dans les activités culturelles et dans le Français Langue de Scolarisation.

On note que les termes de « désegrégation » et de « circulation » font écho au concept d' « inclusion » et que le projet artistique apparait comme un potentiel facteur facilitant la reconnaissance d'un patrimoine linguistique existant, qu'il s'agit de positionner comme central et de patrimonialiser.

\subsection{Les EANA : un public cible mais « empêché »?}

Pour la structure, ces enjeux de patrimonialisation et de dialogue avec le territoire sont également importants. MA fait partie des institutions culturelles publiques décentralisées, plus précisément des 74 « scènes nationales ${ }^{10}$ » de France. Ces scènes possèdent 3 missions : soutenir la création contemporaine en finançant des résidences et en mettant à disposition leurs lieux et leurs équipes, diffuser des spectacles pluridisciplinaires issus des arts vivants et participer activement à l'éducation artistique du territoire dans lequel elles sont implantées, dans une visée de « démocratisation » et de faciliter l'accès à la culture pour les populations. Depuis 2015, la France et plus largement l'Europe, multiplient les initiatives visant à mobiliser ces instances dans l'accueil des populations migrantes. Les très nombreuses propositions doivent aussi faire évoluer les représentations sociales sur les migrations, comme le souligne Gruson, chargé d'examiner «la politique d'accueil des réfugiés mise en place par le gouvernement à partir de septembre 2015, dans sa dimension culturelle mais aussi linguistique, et d'élaborer des préconisations pratiques. » Il engage les acteurs à mener un « travail réciproque [...] vers la société dans son ensemble pour contribuer à changer les regards et combattre les stéréotypes et la xénophobie »(Gruson, 2017 : 173).

Parmi les appels à projets visant à favoriser l'intégration des « migrants » ou des « réfugiés » et à faciliter leur « apprentissage de la langue française », citons l'action lancée en 2015 par le ministère de la Culture et de la Communication qui a largement inspiré Parlemonde :

10. Information disponible sur https://www.scenes-nationales.fr/label/. [Page consultée le 2 octobre 2020.] 
Langue maternelle pour les uns, langue du pays d'accueil pour d'autres, la langue française permet la communication entre tous, sa maitrise étant une composante essentielle du vivre ensemble. [...] Il est donc nécessaire, dans une perspective de réduction des inégalités face à la langue française, d'en faciliter l'accès pour toutes les personnes chez qui les mécanismes de relégation comme de non-participation à la vie sociale produisent une insécurité linguistique, qui est souvent culturelle. Qu'il s'agisse de développer les compétences à l'écrit ou à l'oral, de personnes scolarisées en France ou de permettre à des migrants allophones de communiquer en français, à l'oral et à l'écrit, les pratiques culturelles et artistiques, de même que le contact avec les œuvres et les documents originaux constituent un levier d'action efficace pour l'appropriation du français. En retour, celle-ci peut favoriser l'accès à l'offre culturelle. (Ministère de la Culture et de la Communication, $2015: 1$ )

Kneubühler souligne dans son rapport d'évaluation de cette action que « les actions les plus pertinentes [...] sont précisément des actions où, à l'évidence, la coopération entre professionnels de la culture et formateurs linguistiques a été particulièrement bien pensée » (Kneubühler, 2018). On note que 2 discours sont présents : l'un plaçant les pratiques artistiques comme un «levier » d'accès à la langue - à la fois langage, culture et patrimoine -, cela dans un mouvement à sens unique : l'étranger (dont l'accès à la culture serait empêché) apprend notre langue avec ou par les arts, avec des artistes mais accompagnés de «formateurs linguistiques », puis accède à notre culture ; et l'autre discours, rappelé par Gruson, s'appuyant sur les recommandations du Conseil de l'Europe qui pose «la question du rôle de la culture et des arts dans les processus d'accueil et d'intégration des migrants et dans le changement des représentations sur l'immigration » (Gruson, 2017 : 173). Il s'agit ici d'un mouvement de déplacement réciproque dans une visée de dialogue interculturel, inscrit parmi les priorités du COE. Or, selon lui :

Cette approche est rarement revendiquée en France. En particulier, s'agissant des migrants, tout se passe comme si ces populations n'avaient pas de culture et devaient, par l'apprentissage du français notamment, acquérir nos valeurs culturelles. (Gruson, 2017 :173)

Gruson souligne que les institutions culturelles, ayant une mission d'éducation, sont dans une situation complexe reposant sur des traditions d'exclusion de l'altérité. La conception dominante de la langue est celle d'outil, de moyen de communication, mais c'est également un patrimoine culturel à défendre permettant l'accès aux savoirs et aux œuvres produites et exposées par ces institutions culturelles. Ces dernières font l'objet de nombreux débats quant à la nécessité de, selon les termes de Bonniel : «sortir de [leur] auto-centration et d'entrer (plus exactement de revenir) en dialogue avec d'autres mondes sociaux, d'autres réalités institutionnelles, d'autres pratiques professionnelles, des usagers non définis par leur seule adhésion à la foi culturelle » (Bonniel, 2007 : 24). Cela induit des politiques fortes de « démocratisation » et d'accessibilité de l'art et de la culture. Dans le contexte migratoire actuel, elles témoignent d'une volonté de respecter les « droits culturels » des publics, selon cette définition de l'UNESCO « le droit des peuples à leur propre patrimoine culturel, de même que celui de participer au patrimoine culturel de l'humanité» (UNESCO, 2010 : 247). Des initiatives se 
multiplient en direction des «migrants » : offre tarifaire intéressante voire gratuité, propositions de médiations variées, livrets en « français facile » etc. Ces initiatives assimilent les personnes nouvellement arrivées à la fois à un public potentiel à séduire et au groupe des publics « empêchés », expression euphémisante consacrée pour désigner les milieux populaires ou défavorisés. Cette formule de «public empêché » est régulièrement remise en cause par certains chercheurs et professionnels du secteur, comme le souligne Bordeaux:

Dire qu'il n'y a pas de public spécifique, c'est d'abord soutenir qu'il n'y a pas un type d'œuvre d'art ou un type d'artiste prédisposéà s'adresser ä des publics particuliers, quand bien même ceux qui interviennent à l'hôpital, dans une maison d'arrêt, peuvent éventuellement être amenés à concevoir des propositions en fonction du contexte ou des personnes rencontrées. C'est donc refuser tout rapport d'assignation entre œuvres et publics. C'est aussi défendre, préserver, faciliter la possibilité pour chacun de construire une relation autonome, souveraine, à l'œuvre d'art, quelles que soient les spécificités de sa condition. (Bordeaux, 2007: 19)

Pourtant, la représentation sociale du «public empêché » perdure. Le récent rapport commandé par le ministre la Culture à Benjamin Stora intitulé «Culture et migrants », remis en février 2019, en témoigne. On lit notamment que : « l'orientation [des politiques] doit rester liée à l'affirmation d'un accès d'une population empêchée à ses droits culturels » (p. 9); ; il convient de limiter les dispositifs spécifiques et de privilégier une aide à l'accès aux dispositifs de droit commun ou une intégration de ces publics dans les politiques visant les publics éloignés de la culture» (p. 9) ; enfin, au sujet de la qualité artistique des dispositifs mis en place, l'auteur recommande que : « la qualité artistique des interventions peut être un critère mais non la recherche d'une excellence artistique, [...] afin d'éviter une confusion entre des dispositifs à objectifs culturels et des dispositifs à objectif sociaux » (p. 8). Il y aurait donc une partition des arts et de la « qualité artistique » en fonction de la cible. Si la phrase « Les migrants doivent être à la fois considérés comme destinataires de produits culturels mais également comme potentiels créateurs d'une offre culturelle » vient à la fin de cette même page, l'adjectif « potentiel » et le déterminant indéfini « une » relativisent ce propos. Par ailleurs, l'auteur du rapport mentionne que "l'accès le plus rapide possible à l'apprentissage du français ", est la « principale revendication des associations ${ }^{11}$ ", et que cela doit " être pris en compte » par les autorités (p. 8). Il semble que, dans les représentations des acteurs du terrain (« les associations »), la langue précèderait et conditionnerait l'accès à la culture.

C'est dans ce continuum global de l'obstacle et de l'empêchement: barrière de la langue, difficulté et vulnérabilité (linguistique, scolaire ou sociale) des « migrants » reposant sur une vision ethnocentrée et monolingue des institutions, mais également dans un contexte de volonté interinstitutionnelle d'inclusion des populations allophones déplacées qu'intervient Parlemonde. II a été ainsi baptisé pour faire écho d'une part au multilinguisme du territoire (le Pays de Montbéliard, et par

11. Les associations que l'auteur évoque ici sont celles qui font partie des institutions culturelles ou qui sont leurs partenaires privilégiés. 
extension la France, le monde), et d'autre part à une vision plurielle des langues comme vecteur de rencontre interculturelle créatrice. Par paronomase, son nom se réfère aussi à " parlement » selon la proposition de Charlotte Lagrange ${ }^{12}$, membre de l'équipe artistique qui a créé la pièce Sédiments avec des lycéens allophones lors de la première édition du festival.

\section{Créer c'est relier}

Programme de résidences artistiques monté en coopération, Parlemonde fait le pari de la création comme moteur de reliance au sens « activant » que Morin définit ainsi :

La notion de reliance, inventée par le sociologue Marcel Bolle de Bal, comble un vide conceptuel en donnant une nature substantive à ce qui n'était conçu qu'adjectivement et en donnant un caractère actif à ce substantif. «Relié » est passif, « reliant » est participant, « reliance » est activant. (Morin, $2004:$ 239)

En effet, pour ces concepteurs, partager l'acte de création peut permettre à la fois de relier les EANA à une nouvelle langue entrant dans leur vie, à un nouvel environnement social mais plus largement à un nouveau territoire dont l'exploration dépend de l'opportunité et de la richesse des liens sociaux (Pesqueux, 2009) et qu'il s'agit d'habiter ensemble. Déségrégation et accessibilité sont donc au cœur de Parlemonde, inscrivant les partenaires dans une volonté proactive d'inclusion de tous dans la vie culturelle, reprenant les principes du dialogue interculturels tel que décrits en 2008 dans le Livre blanc sur le dialogue interculturel de la COE, ou les Repères pour un dialogue interculturel de Billerey et Hatzfeld, ouvrage publié en 2010 par le ministère de la Culture et de la Communication.

\subsection{Principes de montage du projet partenarial}

Initié lors de l'année scolaire 2016/2017 en coopération entre MA et le Rectorat de l'académie de Besançon, en particulier avec l'équipe du CASNAV, Parlemonde se déroule principalement (mais pas exclusivement) dans des établissements scolaires (écoles, collèges et lycées) et sur le temps scolaire. Les résidences sont comprises entre 60 et 100 heures d'intervention avec les artistes dans les classes. La présence des artistes à l'école n'est pas un fait nouveau et l'équipe de MA dispose même d'un service dédié à ces actions. Cependant, la collaboration école/artiste s'effectue le plus souvent lors de rencontres ponctuelles ou de projets spécifiques qui s'inscrivent dans le cadre d'un cours disciplinaire de la classe ordinaire (arts plastiques, musique ou lettres), d'un atelier artistique ou d'une option (théâtre, danse, musique). Si les collaborations favorisant les pratiques artistiques sont nombreuses et les invitations institutionnelles pour leur développement de plus en plus fréquentes,

12. Nous ne rendons pas anonymes dans cet article les références aux artistes et à leurs œuvres conçues dans le cadre de Parlemonde, ces œurres étant publiques et produites au même titre que le reste de leur travail. Nous rendons anonymes les données que nous avons suscitées, principalement les discours tenus lors des entretiens semi-directifs ou les travaux d'élèves, préparatoires aux œuvres (extraits de copies, textes ou commentaires divers, messages, etc.). 
notamment suite à l'instauration des parcours d'éducation artistique et culturelle (PEAC) $)^{13}$ devant être documenté par chaque élève tout au long de sa scolarité, elles ne sont pas systématiques sur le terrain et n'entrent pas dans les curriculums en tant que telles. Elles sont mêmes parfois vécues comme des injonctions.

Dans le cas qui nous occupe, chaque enseignant s'est porté volontaire pour engager ses élèves dans Parlemonde. La majorité a répondu à l'invitation du CASNAV. Moins de la moitié connaissait les activités de créations participatives de la structure culturelle. Une suite de contacts s'établit dans lesquels sont explorées les envies de chacun, notamment en ce qui concerne la discipline artistique. C'est enfin la structure qui propose les artistes qu'elle engage et produit, après des échanges évoquant le contexte de création, les modalités d'accueil, de contrat et la rédaction d'une « note d'intention » traçant les grandes lignes du projet de chaque artiste. Cette note d'intention est ensuite présentée aux enseignants et éventuellement ajustée en fonction des informations échangées.

\subsection{Enjeux didactiques pour l'école}

La volonté commune des concepteurs de Parlemonde s'appuie sur l'hypothèse de compétences interculturelles et plurilingues importantes (Abdallah-Pretceille, 1999) développées dans un cadre de mobilité ou migration (Moro, 2002, 2010, 2015) et sur l'hypothèse du projet artistique public comme booster de l'apprentissage de la langue française cible (Pierra 2006 ; Schmidt 2006 ; Auger, 2007 ; Clerc et al 2007 ; Aden, 2009, 2013, 2016 ; Eschenauer, 2018). Les équipes du CASNAV, incluant les enseignants des dispositifs UPE2A dans lesquels est scolarisé l'essentiel des participants de Parlemonde, parient sur le fait que les processus et dynamiques de co-création engagés lors des périodes de résidence des artistes aux côtés des EANA transformeraient leurs pratiques (quel que soit leur champ professionnel) et valoriseraient simultanément les compétences des EANA aux yeux de tous (élèves, parents, équipes éducatives et artistiques, public).

Cette conception est également nourrie par les recherches en sciences de l'éducation qui ont montré que la pédagogie de projet accélérait, voire révélait, les compétences des élèves alors même qu'elles étaient invisibles ou invisibilisées par des tâches scolaires ordinaires. Pourtant, monter un tel projet sur le temps de l'école et malgré le soutien du Rectorat ne va pas de soi. Chrifi-Alaoui écrivait en 2009 que « le projet artistique appartient à une catégorie particulière. L'art est un terme et même un domaine qui fait encore peur. On veut l'éloigner de l'école par crainte du désordre ou pire par craindre de perdre son temps » (p.27). En classe ordinaire en effet, les pratiques artistiques restent à la marge, le sont de plus en plus à mesure que les examens s'approchent et où les exigences scolaires s'accroissent. S'il persiste des activités dites artistiques, elles sont le plus souvent ponctuelles et leur finalité est d'accomplir une tâche mobilisant la « créativité » des élèves (conception d'une exposition, présentation aux parents ou à des pairs de textes écrits en cours de français puis joués, restitution de

13. Information disponible sur http://eduscol.education.fr/cid74945/le-parcours-education-artistique-culturelle. html. [Page consultée le 2 octobre 2020.] 
la chorale, etc.). Elles sont le plus souvent conduites sans artiste et prétextes à une co-intervention entre enseignants de disciplines différentes. Plus fréquentes dans le champ de l'enseignement adapté ou spécialisé (dispositifs de lutte contre le décrochage scolaire ou d'accueil des élèves en difficulté ou en situation de handicap), elles s'inscrivent alors dans une démarche de «pédagogie du détour » et sont envisagées comme un vecteur de remobilisation des élèves en difficulté d'apprentissage, ce que Henri-Panabière et al. nomment la pédagogie du « détour pour un retour ». De plus, un pouvoir de « réparation symbolique » agissant sur la personnalité des élèves concernés (Bonnéry et Renard, 2013 : 144) leur est souvent attribué.

\subsection{Enjeux pour la structure artistique}

Pour la scène nationale, Parlemonde est une première dans un tel contexte sociétal et avec de tels participants. Bien que familière des projets de création participative en milieu scolaire, MA connait avec Parlemonde sa première expérience de coopération d'ampleur qui dépassera la durée d'une saison et deviendra un festival en biennale qui connaitra en 2021 sa $3^{\text {ème }}$ édition. C'est donc un investissement important tant dans l'implication des équipes artistiques et techniques que financièrement puisque la structure culturelle supporte l'essentiel des coûts. Voici un tableau récapitulatif qui présente les œuvres créées ${ }^{14}$ en 2017 et 2019 avec 250 participants et une quinzaine d'artistes européens:

Tableau 1 : Récapitulatif des créations Parlemonde

\begin{tabular}{|c|c|c|c|c|}
\hline \multirow{4}{*}{ Parlemonde 1 } & Artistes & Titres & Disciplines & Participants \\
\cline { 2 - 5 } & Sébastien Fayard & (Dé)formations & Photographie & CM2 \\
\cline { 2 - 5 } Mai 2017 & Thomas Boichard & Déviation & Musique & $\begin{array}{c}\text { MNA (hors temps } \\
\text { scolaire) }\end{array}$ \\
\cline { 2 - 5 } & David Subal & $\begin{array}{c}\text { Orientations (Un par- } \\
\text { tage des chemins) }\end{array}$ & $\begin{array}{c}\text { Installation, exposi- } \\
\text { tion et performance }\end{array}$ & UPE2A lycée \\
\cline { 2 - 5 } & Wil Mathijs & Becoming & Vidéo & UPE2A collège \\
\cline { 2 - 5 } & Frédéric Dumond & $\begin{array}{c}\text { Le Bruissement des } \\
\text { langues }\end{array}$ & $\begin{array}{c}\text { Poésie et perfor- } \\
\text { mance }\end{array}$ & UPE2A lycée \\
\cline { 2 - 5 } & Charlotte Lagrange & Sédiments & Théâtre & UPE2A lycée \\
\hline
\end{tabular}

14. D’autres éléments ainsi que des images et/ou vidéos sont disponibles sur le site dédié en cours d'actualisation: http://parlemonde.mascenenationale-creative.com 


\begin{tabular}{|c|c|c|c|c|}
\hline \multirow{4}{*}{ Parlemonde 2 } & $\begin{array}{c}\text { Cédric Charron \& } \\
\text { Annabelle Chambon }\end{array}$ & $\begin{array}{c}\text { Service d'Action Cho- } \\
\text { régraphique }\end{array}$ & Danse/ Performance & $\begin{array}{c}\text { Tout public (hors } \\
\text { temps scolaire) }\end{array}$ \\
\cline { 2 - 5 } Mai 2019 & Thomas Boitage & $\begin{array}{c}\text { Musique et arts plas- } \\
\text { tiques }\end{array}$ & $\begin{array}{c}\text { CM2 + CM2 et CE2 } \\
\text { pour le volet illustra- } \\
\text { tion graphique }\end{array}$ \\
\cline { 2 - 5 } & $\begin{array}{c}\text { David Subal \& Clara } \\
\text { Cornil }\end{array}$ & $\begin{array}{c}\text { D'ailleurs \& Carte } \\
\text { d'identités }\end{array}$ & $\begin{array}{c}\text { Installation, exposi- } \\
\text { tion et performance }\end{array}$ & $\begin{array}{c}\text { UPE2A Cité scolaire } \\
\text { (collège et lycée) }\end{array}$ \\
\cline { 2 - 5 } & Jorge Picó & Sauver les mots & Théâtre & UPE2A collège \\
\cline { 2 - 5 } & $\begin{array}{c}\text { Renaud Diligent \& } \\
\text { Maya Boquet }\end{array}$ & $\begin{array}{c}\text { Un Banquet } \\
\text { Cédric Orain }\end{array}$ & $\begin{array}{c}\text { Bibliothèque Hu- } \\
\text { maine Internationale } \\
\text { (BHI) }\end{array}$ & $\begin{array}{c}\text { UPE2A lycée + } \\
\text { classe de CAP }\end{array}$ \\
\cline { 2 - 5 } & $\begin{array}{c}\text { Écriture/ } \\
\text { performance }\end{array}$ & $\begin{array}{c}\text { Tout public (hors } \\
\text { temps scolaire) }\end{array}$ \\
\hline
\end{tabular}

D’un point de vue méthodologique, les artistes ont été formés par le CASNAV en amont des résidences sur la question des plurilinguismes et de l'allophonie. Ils sont libres d'engager leur résidence dans la (ou les) langue(s) qu'ils souhaitaient, tous ne sont pas des francophones natifs mais tous sont plurilingues comme le montre le tableau suivant:

Tableau 2 : Répertoires linguistiques des artistes ayant travaillé en milieu scolaire

\begin{tabular}{|c|c|c|}
\hline & Langues premières & $\begin{array}{c}\text { Autres langues (par ordre de com- } \\
\text { pétence estimée) }\end{array}$ \\
\hline Artiste 1 & Français & Allemand, anglais \\
\hline Artiste 2 & Français & Anglais, espagnol \\
\hline Artiste 3 & Néerlandais flamand & Anglais (LS), français \\
\hline Artiste 4 & Français & Anglais, espagnol \\
\hline Artiste 5 & Français & $\begin{array}{l}\text { Latin, grec ancien, anglais, grec } \\
\text { moderne }\end{array}$ \\
\hline Artiste 6 & Allemand (Autriche) & Anglais (LS), français, latin \\
\hline Artiste 7 & Catalan, espagnol & Anglais, français \\
\hline Artiste 8 & Français & Allemand, anglais \\
\hline
\end{tabular}

Le choix des langues de travail s'est fait le plus souvent spontanément et principalement en fonction des interactions avec les participants. Les artistes de langue maternelle française les ont conduites en français exclusivement, les autres ont utilisé toutes les langues de leur répertoire, souvent pour traduire ou développer une consigne.

Le projet artistique est resté un espace de création donc peu contraint. Deux points de vigilance ont cependant été abordés dès les premiers échanges entre les différents partenaires : permettre aux participants de s'exprimer dans les langues de leur choix, et cela sans assignation particulière et avec la possibilité d'en changer à tout moment ; évacuer les entrées artistiques reposant purement sur des considérations biographiques de type «témoignages » ou documentaire visant à faire partager 
les parcours de migrations et autres traumatismes vécus au cours de celle-ci au public, écueil parfois présent dans la création théâtrale contemporaine (Carré, 2017).

\subsection{Place et profils linguistiques des élèves participants}

Les participants ont co-écrit, monté, joué les œuvres dont le processus créatif avait été imaginé par les artistes dans leur note d'intention. En cela, les élèves ont été co-auteurs, co-créateurs à des degrés divers de participation selon les sensibilités de chacun, mais ils n’ont pas mis en scène ou chanté ou exposé de textes pré-écrits ou pré-imaginés pour eux. Ils portent leur parole, négociée, travaillée collectivement et individuellement, dont les fonctions esthétiques et symboliques sont à la fois conscientisées et valorisées. Cette parole s'exprime dans de nombreuses langues. Pour en avoir une idée, voici les langues familiales déclarées par participants des résidences scolaires suivies ${ }^{15}$, sachant qu'elles composent mais ne reflètent pas de manière exhaustive les répertoires linguistiques des élèves. Sur les 167 élèves documentés : 90 déclarent au moins deux langues familiales. On dénombre 37 langues déclarées (mais 35 distinctes) et 1 participant dont la langue première n'est pas renseignée ; 10 participants pour lesquels le français est cité en premier dans les langues familiales, 26 pour lesquels le français est cité comme seconde langue. Aucun des 167 élèves interrogés ne se définit comme monolingue (y compris les locuteurs natifs de France).

Tableau 3 : Langues familiales déclarées par les élèves

\begin{tabular}{|c|c|c|c|}
\hline Langues familiales & Nombre de locuteurs & Langues familiales & Nombre de locuteurs \\
\hline Arabe & 68 & Ourdou & 3 \\
\hline Français & 36 & Malinké & 2 \\
\hline Italien & 21 & Dioula & 2 \\
\hline Albanais & 14 & Swahili & 2 \\
\hline Espagnol & 12 & Somali & 2 \\
\hline Portugais & 10 & Sylheti & 1 \\
\hline Bambara & 8 & Wolof & 1 \\
\hline Lingala & 7 & Amharique & 1 \\
\hline Turc & 7 & Baoulé & 1 \\
\hline Serbo-croate & 7 & Kimbundu & 1 \\
\hline Peul & 5 & Kirghize & 1 \\
\hline Shimaoré & 5 & Mandarin & 1 \\
\hline Bengali & 4 & Soussou & 1 \\
\hline Soninké & 4 & Tchèque & 1 \\
\hline Persan & 3 & Tzigane & \\
\hline Kabyle & 3 & Diakhanké & \\
\hline
\end{tabular}

15. Sur les 251 participants au total, les chiffres présentés ici concernent les élèves du secondaire (collège et lycée) soit 167 élèves ayant participé à 7 projets. 


\begin{tabular}{|c|c|c|c|}
\hline Langues familiales & Nombre de locuteurs & Langues familiales & Nombre de locuteurs \\
\hline Russe & 3 & Non référencé & 1 \\
\hline Rif & 3 & & \\
\hline
\end{tabular}

\section{Les pratiques artistiques : du détour au passe-murailles}

Le recours aux pratiques artistiques pour remédier à une situation particulière ou pour maintenir la « cohésion sociale » renvoie à l'idée que l'art, selon la formule de Pignot (2007) serait « passe-muraille » et permettrait de renverser, voire de soigner les fractures sociales de nos sociétés. Dans notre cas, les arts ont surtout permis de faire œuvre et de faire entendre la voix des élèves au sens propre comme au figuré, favorisant leur inclusion sociale.

\section{1. Évolution des représentations à propos des langues des participants}

La variété des langues et des contextes de leur utilisation a suscité nombre d'échanges et d'interrogations, tant du côté des artistes, peu habitués à co-créer avec des jeunes allophones (2 en avaient l'expérience), que du côté des participants, peu habitués à travailler avec des artistes, encore moins dans un cadre scolaire, et surpris d'être invités à dévoiler, et à utiliser, leurs langue(s) première(s). Nous ne développerons pas ici les étapes du processus de création ni celles qui ont conduit, le cas échéant, au changement de point de vue quant aux langues des participants. Cependant nous pouvons évoquer les moments de médiation que nous avons conduits afin de répondre à certaines questions des artistes ou d'en faire germer d'autres. Cette fonction de médiation, initialement imprévue (Sérusclat-Natale et Marzin, 2020) a été très importante pour conscientiser, auprès des acteurs concernés par l'expérience esthétique (participants au sens large et publics), les représentations qu'ils avaient des langues et des cultures, puis d'explorer leurs propres origines.

\subsubsection{Pour les artistes}

Aucun artiste n'a demandé comment communiquer avec les élèves, mais tous se sont questionnés sur les possibilités d'accès à l'écrit. On a constaté dans les entretiens de fin de projet menés avec eux que le niveau de compétences en français des élèves était généralement oublié, comme en témoigne artiste 8 ici :

D : Avais-tu déjà travaillé avec les élèves allophones?

Artiste 8 : J'ai déjà travaillé dans des conditions compliquées avec des jeunes... heu... tu vois, de... issus de... de tu vois... en galère sociale et en rupture scolaire et enfin voilà dans des conditions compliquées heu... voilà... mais ce type de public avec heu différentes langues... mais en même temps ils parlaient beaucoup, tous français, hein quand même pour la plupart, il... il y avait quand même peu de vraiment de non... enfin allophones à $100 \%$ et heu... 
Or, dans cette résidence effectuée avec 15 élèves de l'UPE2A, 7 élèves ne s'exprimaient pas du tout en français au démarrage du projet. L'artiste n'en garde pas le souvenir mais assimile les EANA aux publics « empêchés » avec lesquels il a déjà travaillé auparavant. Pour certains artistes, le plurilinguisme des participants les attirait parce que le sujet était actuel (le plurilinguisme étant associé au thème des migrants à leurs yeux, l'un a d'ailleurs employé l'expression « me frotter à ça ») pour d'autres c'était un défi à relever dans le sens d'un critère supplémentaire d'« excellence » du projet. Au fil des résidences, les questions se sont portées plus largement sur comment ils pouvaient accéder à l'imaginaire des jeunes, à leurs regards sur le monde et à ce qu'ils avaient envie d'exprimer. Il ne s'agissait plus de communiquer mais plutôt de s'exprimer. Les langues employées n'étaient pas un obstacle, le cadre scolaire l'a été, comme on le voit dans les extraits d'entretien suivants :

\section{Entretien semi-directif avec Artiste 5 post Parlemonde 1}

D: Vois-tu une/ des différences entre les publics scolaires avec lesquels tu as déjà travaillé et les élèves allophones de cette année?

Artiste 5 : Je ne vois pas tant de différence. À part... non non vraiment j'en ai pas vu. À part cette histoire d'efficacité plutôt que dans la création, la question de la rentabilité [...] Une grosse exigence scolaire chez certains, chez d'autres moins. Plus largement, plus d'exigence scolaire qu'avec les autres publics avec qui j'ai travaillé. [...]

D : Tu pourrais décrire les participants?

Artiste 5 : Volontaires, prêts à expérimenter tout sans comprendre forcément - quels étaient les enjeux je veux dire - mais dans le désir, le plaisir de voir où ça menait. Des doutes, forcément mais émis ou gardés avec discrétion, mais qui se font sentir, c'est normal. Une vraie volonté, enfin l'histoire c'est qu'on y va quoi! Quel que soit ce qu'on leur propose, ils y vont quoi ! Il y aurait pratiquement plus de volonté chez eux que dans les groupes ordinaires.

\section{Entretien semi-directif avec Artiste 7 post Parlemonde 2}

Artiste 7 : Au débutj'ai pensé « ils ne peuvent pas » parce qu'il y avait certains conflits entre eux qui disaient qu'ils n'étaient pas dans des conditions de faire du théâtre, ils ne formaient pas un groupe tu vois, et ils étaient petits. À mon avis le processus ils ont grandi pendant les répétitions et ils ont compris que... il faut dépasser ça tu vois. Au-delà de ça, c'est des gens qui ont un vécu personnel, le fait de devoir s'exprimer dans une langue et puis d'un coup dans une autre heu, ça les rend plus forts tu vois. Mais au début effectivement je me suis dit « ça va être trop quoi », parce que... ils ont déjà heu... déjà la situation un peu spéciale mis de côté un peu ghetto quand même, ça fait que entre eux, ils ont des problèmes pour s'accepter comme un groupe, pour s'aimer comme un groupe, et mais une fois que j'ai compris qu'ils pouvaient dépasser cela... ils comprennent quoi. Ils comprennent. Ils comprennent bien quoi [...] Mais heu... au début on a tous peur hein, même le metteur en scène hein... On va y pas arriver, on 
ne sait pas trop où on va aller. [...] C'est vrai qu'humainement le groupe avec les allophones c'est le plus fort quoi.

D : Humainement? Pourquoi tu dis ça?

Artiste 7 : au niveau des réactions, des palpitations, des demandes, [...] ça palpitait plus que dans les autres groupes, et ça c'est très beau. C'est très important. Parce que si ça palpite ça veut dire c'est vivant derrière, y a des vrais conflits des vraies passions, le théâtre il est fait de ça tu vois. Dans les autres groupes il y a eu moins ça; il y a eu beaucoup moins ça.

Ces extraits montrent que l'allophonie et le plurilinguisme, initialement considérés comme un sujet auquel il faudra «se frotter », ont fait écran. La difficulté ressentie venait plutôt à la position de vulnérabilité de l'artiste lui-même au démarrage d'une création. Cette vulnérabilité s'efface dans et par le travail artistique et ce processus révèle d'autres obstacles plus systémiques comme la peur du désordre du côté des enseignants et le souci de rentabilité du temps scolaire.

À ce sujet, Artiste 7 déclare :

Même si après on a besoin des règles de cadrer tout ça oui, mais quand même, certains profs n’ont pas profité du désordre. Le théâtre est lié au désordre. Le désordre c'est très important, le chaos s'il évolue, s'il est aimé, il est très important dans un processus d'éducation, c'est une métaphore de la vie, la vie on ne la contrôle pas tout le temps. Il faut décontroler ${ }^{16}$ les groupes.

\subsubsection{Pour les élèves}

Nous appuyons notre propos sur les mots utilisés par les participants dans les questionnaires de fin de projet, ou dans les entretiens semi-directifs ou dans nos notes de terrain. Pour l'écrasante majorité des élèves participants à Parlemonde, ce festival était la première occasion de collaborer avec un artiste. Certains ont éprouvé quelques difficultés à s'adapter à ces activités artistiques dans la mesure où elles ne faisaient pas partie de leurs représentations de l'école « sérieuse », et du travail scolaire « pour progresser ». Cela a particulièrement été le cas des lycéens, soumis à une forte pression scolaire. On peut d'ailleurs se demander si ces représentations des pratiques artistiques comme « loisirs » sont les leurs, ou si elles témoignent d'une intériorisation des représentations de certains enseignants ou adultes considérant les arts comme une récompense divertissante. Armagnague et al., en 2019, parlent d'une «scolarité loisirs » en abordant ces pédagogies, ce qui montre que cette représentation des pratiques artistiques demeure. Il a donc fallu effectuer ponctuellement des médiations pour encourager les jeunes à poursuivre le projet et les rassurer sur les modalités de rattrapage ou les dispenses des cours manqués. Ces médiations, parfois devant désamorcer des tensions, ont été saluées en fin de projet par les lycéens qui déclarent s'être sentis « entre adultes » (faisant référence à l'horizontalité des relations qui s'était installée par les pratiques artistiques et à la liberté

16. Artiste 7 est hispanophone. En espagnol « descontrolar » est un verbe qui veut dire « perdre le contrôle». 
de s'exprimer). Ils ont également très souvent relevé qu'ils avaient fortement apprécié « être encouragés » par les enseignants, les artistes et les équipes de production, ce qui les a rendus « forts ». Enfin, concernant l'appropriation de la langue française, les EANA disent qu'ils ont progressé, notamment à l'oral : « J'ai appris le français, j'ai appris que j'étais capable. » Pour quelques-uns, Parlemonde a été l'occasion de s'exprimer en français pour la première fois, profitant d'un effet de groupe et d'un effort particulier fait « pour l'artiste » ou « pour notre troupe ». Ils déclarent par exemple : « Oui ça m’a aidée car tout le monde a parlé français donc pour parler avec eux on parle aussi français »; " Avant je ne savais pas m'exprimer mais après le spectacle je m'exprime et je reste plus dans mon coin toute seule. Maintenant je parle plus avec les gens et je suis moins timide. » L'envie de «se lancer » vient aussi de l'approche poétique de la langue comme moyen d'expressivité subjective et non plus comme instrument de communication scolaire, comme l'exprime cet élève : "Dans le projet on a mélangé les langues de tous et je trouve que tout se complète. Un peu. J'ai aimé parler bengali et anglais. Mais maintenant j'aime beaucoup le français et je ne parle plus anglais alors que c'est la langue que tout le monde veut me parler. Aujourd'hui, je me lance dans le français. Je travaille mon accent, le français c'est une langue comme de la musique, ça ressemble à la musique des oiseaux, c'est rapide et dans tous les sens. »

Certains relient leur implication dans Parlemonde et leurs bons résultats au DELF. Pourtant, la majorité des participants déclare que faire partie du projet ne les a pas particulièrement aidés dans leur «métier d'élève ». Si la « rentabilité scolaire » est une inquiétude pendant le temps de résidence et parce que la création s'inscrit dans un temps long, non séquencé comme le temps scolaire et plus opaque (parfois on ne sait pas où l'on va), après le projet, les élèves n'expriment pas d'insatisfaction particulière ni n'affirment avoir perdu leur temps. Au contraire, tous (sauf 2 élèves) voudraient recommencer. Alors que j'interrogeais un groupe de lycéens sur ce que je percevais comme un paradoxe (vouloir recommencer alors que cela ne leur a pas « servi » dans leur métier d'élève), l'une d'entre eux, après quelques secondes de silence, m’a répondu : «Aujourd'hui, maintenant, quand je marche : j’ai la tête haute. » Cette élève n'est pas un cas isolé. Les propos relatifs à la hausse de l'estime de soi et au sentiment d'être ou de se sentir (enfin) capable, ont été nombreux et les élèves associent, dans leurs réponses, ce sentiment de mieux-être à leur réussite scolaire.

\subsection{L'importance de l'exposition publique}

Nous n'aborderons pas ici les traces de réception par le public mais plutôt les propos des élèves participants au sujet du temps des représentations publiques. Aboutissement valorisant du travail engagé, le temps du festival est un temps fort pour tous. C'est aussi le point culminant du stress ressenti et de l'envie de bien faire, et le dernier déclencheur du passage en français pour de nombreux participants. L'un d'eux, âgé de 12 ans, l'exprime ainsi : « Finalement j'ai pas utilisé mon arabe. Mme $X$ m'a laissé le choix mais je n'avais pas envie de passer mon temps à traduire, j'ai préféré qu'on m'entende en français. » Une autre, lycéenne, a choisi de traduire en français ses propos enregistrés en arabe lors de la résidence et de proposer les deux langues au public avançant: « Je pense que le 
public il va parler français alors je veux être sure qu'il m'écoute, j'ai des choses à lui dire. » Il s'agit pour eux d'adresser une parole, non pas seulement de parler ou d'être visibles dans un travail créatif dont ils n'auraient pas élaboré le message et le sens. Ainsi, pour que les expériences artistiques soient profitables, la co-création et l'exposition publique incluant des interactions sont deux aspects majeurs. Dans ce travail commun s'est installée une relation et de la qualité de celle-ci, de son intensité, dépend l'œuvre finale et sa présentation publique. Cela rappelle les termes de Moro :

C'est le processus social, culturel, identitaire, dans lequel sont [les EANA] qui est vulnérabilité mais il peut devenir créativité au sens où on peut faire quelque chose avec ces différences à partir du moment où on les reconnait, on les inscrit, on les valorise, où elles ne restent pas hors cadre, hors école. (Moro, 2017)

On peut aller plus loin et dire qu'elles ne doivent pas rester hors cité, et que l'aspiration existentielle selon les mots du poète Francis Ponge, le « je parle et tu m'entends donc nous sommes » est très importante pour les EANA. Selon Artiste 7, ce sentiment procède du travail artistique : "Qui je suis?" demande le théâtre, le théâtre te fait dire "je suis par rapport à quelqu'un". On a pas mal creusé le "qui je suis" avec ce groupe, mon essence, l'essence, mais ton essence c'est le... le rapport à l'autre, face au visage de l'autre et aux mots de l'autre. »

Parmi les performances réalisées par les élèves, 2 ont été faites en interaction directe avec le public ${ }^{17}$, soit sous la forme de "face à face ", soit de performances chez l'habitant. Summum de l'exposition au sens où les participants sont, d'une part, amenés à suivre un protocole artistique abolissant les distances, tant physiques que psychologiques, avec un public d'inconnus, et d'autre part à improviser en fonction des réactions de l'auditoire. Ces projets sont ceux qui ont été préférés par les participants, décrits par de nombreuses expressions superlatives comme une " aventure fantastique », « forte », « incroyable ». Un lycéen explique : « C'est vraiment la prise de risque qui m’a plu, c'est pour ça que je l'ai préféré. »

\section{Conclusion}

Tenter l'expérience de la co-création artistique avec les EANA ne va pas de soi et ne constitue pas un remède miracle aux situations de vulnérabilité rencontrées par les élèves et les enseignants, souvent érigées par le système scolaire lui-même. En revanche, lorsqu'elles sont authentiquement participatives, elles permettent d'inscrire les apprenants dans une relation fondée sur l'intersubjectivité qui autorise à la fois les recours à toutes les langues des répertoires, mais également les erreurs, les essais, en un mot : le risque, comme le synthétise Coïaniz:

17. Il s'agissait du travail mené avec l'artiste David Subal en 2017 et en 2019. Voir http://parlemonde.mascenenationale-creative.com 
Un apprentissage quel qu'il soit (et à bien des égards, plus encore le langage, en cela qu'il vient profondément reconstruire nos repères et notre vision du monde) apparait comme une conquête, un risque (pour l'image de soi, la place qu'on occupe la cohésion parfois difficilement tenue entre nos pratiques, ce qu'on en ressent, ce qu'on en dit et ce que l'on se représente), conquêtes et risques toujours interactionnels donc toujours sociaux. (Coïaniz, 2001 : 120)

Parler et se dire dans une autre langue ne requiert pas qu'enseignement de la grammaire ou du lexique d'usage. Cela requiert avant tout audace, imagination, flexibilité et désir, qualités très présentes chez les EANA que nous avons suivis, et que rend visibles la pratique artistique. Souvent perçues comme inutiles ou peu rentables scolairement, ou au contraire thérapeutiques et vectrices de « réparation symbolique », les expériences de collaboration artistiques ne peuvent s'improviser et se passer de médiations expertes. Elles s'inventent en équipe. Enfin, elles renforcent, par un mouvement de « décentration des habitus » (Pierra, 2006) dont chaque participant (enfants comme adultes, acteurs comme spectateurs) fait l'expérience, et rendent, à la fois possibles et publiques, une reconnaissance sensible et partagée des langues et des cultures qui font l'identité d'un territoire. 


\section{Références}

Abdallah-Pretceille, Martine (1999), L'éducation interculturelle, Paris, Presses Universitaires de France.

Adam-Maillet, Maryse et Maud Sérusclat-Natale (2019), "L'hospitalité, l'école et le sacré, la question des mineurs non accompagnés », Diversité, nº 196 (L’hospitalité n², sous la dir. de Régis Guyon), p. 126-132.

Aden, Joëlle (dir.) (2008), Apprentissage des langues et pratiques artistiques : créativité, expérience esthétique et imaginaire, Paris, Le Manuscrit Universitê.

Aden, Joëlle (2013), « Apprendre les langues par corps », dans Pour un Théâtre-Monde: plurilinguisme, interculturalitéet transmission, Bordeaux, Presses Universitaires de Bordeaux.

Aden, Joëlle (2016), « Créer, innover par le théâtre : pour une pédagogie énactive des langues », dans Isabelle Capron Puozzo (dir.), La créativité en éducation et en formation : perspectives théoriques et pratiques, Louvain-la-Neuve, De Boeck, p. 107-118.

Armagnague, Maïténa, Alexandra Clavé-Mercier, Marion Lièvre et Anne-Claudine Oller (2019), "Les dispositifs de scolarisation des enfants et jeunes migrants en France: des producteurs de "scolarités contraintes" », Cahiers de la recherche sur l'éducation et les savoirs, nº 18, 147-172.

Armagnague, Maïténa, Isabelle Rigoni, Claire Cossée, Catherine Mendonça Dias et Simona Tersigni (2018), Étude sur la scolarisation des élèves allophones nouvellement arrivés (EANA) et des enfants issus de familles itinérantes et de voyageurs (EFIV), Rapport de recherche EVASCOL, disponible sur https://www.defenseurdesdroits.fr/fr/etudes-et-recherches/2018/12/etude-sur-la-scolarisation-des-eleves-allophones-nouvellement-arrives. [Page consultée le 17 août 2020.]

Auger, Nathalie, Olivier Terrades et Richard Talagrand (2007), «Ateliers littérature et arts pour entrer dans le français langue seconde », ELA: Études de linguistique appliquée, vol. 147, n³ 3, p. 339-348.

Auger, Nathalie (2008), "Le rôle des représentations dans l'intégration scolaire des enfants allophones » dans Jean-Louis Chiss (dir.), Immigration, École et didactique du français, Paris, Didier, p.187-230.

Auger, Nathalie (2010), Élèves nouvellement arrivés en classe : réalités et perspectives, Paris, Éditions des archives contemporaines.

Auger, Nathalie (2013), «Continuum/clivage de représentations dans les définitions de la difficulté scolaire en matière de langue(s) », dans Stéphanie Galligani, Sandrine Wachs et Corinne Weber (dir.), École et langues: des difficultés en contextes, Paris, Riveneuve, p. 119-138.

Blanchet, Philippe (2016), Discriminations : combattre la glottophobie, Paris, Textuel.

Billiez, Jacqueline (1985), «La langue comme marqueur d’identité », Revue européenne des migrations internationales, vol. 1, n², p. 95-105.

Bonnéry, Stéphane et Fanny Renard (2013), « Des pratiques culturelles contre l'échec et le décrochage scolaires : sociologie d'un détour », Lien social et Politiques, n 70, p. 135-150. 
Bonniel, Jacques (2007), «Élargir le cercle des connaisseurs », L'Observatoire : la revue des politiques culturelles, n 32, p. 23-27.

Bordeaux, Marie-Christine (2007), "Il n’y a pas de public spécifique », L'Observatoire : la revue des politiques culturelles, n³2, p.19.

Calvès, Anne-Emmanuèle (2009), « "Empowerment” : généalogie d’un concept clë du contemporain sur le développement », Tiers Monde, vol. 200, nº 4, p. 735-749.

Calvet, Louis-Jean (1974), Linguistique et colonialisme: petittraité de glottophagie, Paris, Payot. Carré, Alice (2017), « Récits d'exil en scène », E-Migrinter, n 16, disponible sur http://journals.openedition.org/e-migrinter/941. [Page consultée le 16 août 2020.]

Chrifi-Alaoui, Dalie (2009), «Apprendre le français par l'oblique », Cahiers pédagogiques, n 473, p. 2628.

Clerc, Stéphanie, Claude Cortier, Amandine Longeac et Gérard Oustric (2007), «Place et intérêt des pratiques artistiques, interculturelles dans les dispositifs d'accueil et d'enseignement/apprentissage pour les élèves allophones », ELA: Études de linguistique appliquée, vol. 147, n³, p. 317-328.

Coïaniz, Alain (2001), Apprentissage des langues et subjectivité, Paris, L'Harmattan.

Cummins, Jim (2001), Negociating identities : Education for empowerment in a diverse society, $2^{e}$ éd., Los Angeles, California association for bilingual education. [1e éd., 1996.]

Ebersold, Serge et Maïtena Armagnague-Roucher (2017), «Importunité scolaire, orchestration de l'accessibilité et inégalités », Éducation et sociétés, n³9, p.137-152.

Ennuyer, Bernard (2017), «La vulnérabilité en question », Ethics, Medicine and Public Health, n³, p. 365-373.

Eschenauer, Sandrine (2018), "Créativitê et empathie dans les apprentissages performatifs : vivre et encorporer ses langues », Recherches \& Éducation, hors-série, disponible sur : http://journals.

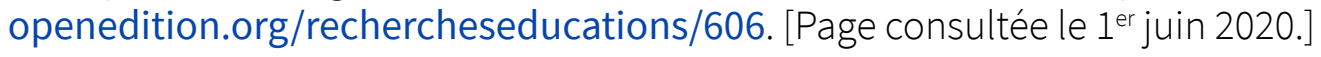

Escudé, Pierre (dir.) (2018), Langues et discriminations, numéro thématique des Cahiers de la LCD, $n^{\circ}$ 7, Paris, L'Harmattan.

García, Ofelia (2009), «Emergent bilingual and TESOL: What's in a name? », TESOL Quaterly, vol. 43, no 2, p. 322-326.

Gavini-Chevet, Christin, Yves Delécluse et Christian Bigaut (2018), Les aménagements d'épreuves d'examens pour les élèves et étudiants en situation de handicap, rapport de l'Inspection générale de l'administration de l'Éducation nationale et de la Recherche, disponible sur https://www. education.gouv.fr. [Page consultée le 2 octobre 2020.]

Goï, Cécile (2015), « Langues et rencontre interculturelle en éducation : loyautés, conflits, autorisations » dans Accueillir l'enfant et ses langues : rencontres pluridisciplinaires sur le terrain de l'école, Paris, Riveneuve. 
Gruson, Luc (2017), «Comment mettre la culture au service des migrants? » Hommes et migrations, vol. $1, n^{\circ} 1316$, p. 170-179.

Henri-Panabière Gaële, Fanny Renard et Daniel Thin (2013), « Des détours pour un retour? Pratiques pédagogiques et socialisatrices en ateliers relais », Revue française de pédagogie, n 183, p. 71-82.

Kneubühler, Michel (2018), Détours \& déclics : action culturelle et langue française, Paris, La passe du vent.

Lüdi, Georges et Bernard Py (1986), Être bilingue, Berne, Peter Lang.

Mestre, Claire (2015), "Parentalité, migration et exil, comment prendre soin des parents ? », Spirale, n०73, p. 206-216.

Métraux, Jean-Claude (2011), La migration comme métaphore, Paris, La Dispute.

Ministère de la Culture et de la Communication (2015), Appel à projets national : "L'action culturelle au service du français », disponible sur https://aaar.fr/wp-content/uploads/2015/06/Appel-aprojets.pdf. [Page consultée le 2 octobre 2020.]

Morin, Edgar (2004), La Méthode, vol. 6, Paris, Seuil.

Moro, Marie-Rose (2002), Enfants d'ici venus d'ailleurs, Paris, Hachette Littératures.

Moro, Marie-Rose (2010), Grandir en situation transculturelle, Paris, Fabert.

Moro, Marie-Rose (dir.) (2015), L'adolescent créatifface aux malaises de la société, Paris, Armand Colin.

Moro, Marie-Rose (2017), Pilotage des dispositifs d'accueil et de scolarisation des élèves allophones nouvellement arrivés: quels parcours d'inclusion pour des élèves en situation de vulnérabilité ?, discours prononcé dans le cadre du Plan national de formation d’Éduscol, disponible sur https://www.canal-u.tv/video/eduscol/intervention_de_marie_rose_pilotage_des_dispositifs_d_accueil_et_de_scolarisation_des_eleves_allophones.3679. [Page consultée le 2 octobre 2020.]

Organisation de coopération et de développement économiques (OCDE) (2008), Élèves présentant des déficiences, des difficultés et des désavantages sociaux, disponible sur http://www.oecd. org/fr/education/scolaire/41058722.pdf. [Page consultée le 2 octobre 2020.]

Organisation mondiale des Nations unies pour l'éducation, la science et la culture (UNESCO) (2010), Investir dans la diversitéc culturelle et le dialogue interculturel : rapport mondial de l'UNESCO, Paris, Éditions UNESCO.

Pesqueux, Yvon (2009), «La notion de territoire », communication présentée au colloque Observatoire économique des banlieues, Propedia, Paris, décembre 2009, disponible sur : https://hal.archives-ouvertes.fr/hal-00479794/document. [Page consultée le 7 novembre 2020.]

Pierra, Gisèle (2006), Le corps, la voix, le texte, arts du langage en langue étrangère, Paris, L'Harmattan. 
Pignot, Lisa (2007), «L'art comme passe-muraille », L'Observatoire : la revue des politiques culturelles, n०32, p. 20-23.

Puren, Christian (s.d.) La pédagogie de projet dans la mise en œuvre de la perspective actionnelle, disponible sur www.christianpuren.com. [Page consultée le 2 octobre 2020.]

Renaut, Alain (2009), Un humanisme de la diversité : essai sur la décolonisation des identités, Paris, Flammarion.

Schmidt, Prisca (2006), « Le théâtre comme art de l'apprentissage de la langue étrangère », Spirales, n०38, p. 93-109.

Sen, Amartya (2009), L'idée de justice, Paris, Flammarion.

Sérusclat-Natale, Maud et Yannick Marzin (2020), « Les jeux de la recherche collaborative : le cas du projet artistique », Recherches en didactique des langues et des cultures, vol. 17, $n^{\circ} 2$, disponible sur: http://journals.openedition.org/rdlc/7712. [Page consultée le 3 août 2020.]

Stora, Benjamin (2019), La culture et les migrants, rapport remis au ministre de la Culture, disponible sur https://benjaminstora.univ-paris13.fr/images/stories/actualites/STORA_Mission_ Rapport_vers_trav.pdf. [Page consultée le 2 octobre 2020.]

Veret, Thomas (2018), « Compte rendu de Pierre Escudé (dir.), "Langues et discriminations", Les cahiers de la $L C D, n^{\circ} 7$ », Lectures, disponible sur http://journals.openedition.org/lectures/32399. [Page consultée le 2 octobre 2020.] 